\title{
Is Penile Shortening Part of Natural History of Peyronie's Disease?
}

\author{
Sudhanshu Chitale", Mohammed Morsey and Krishna Sethia
}

Department of Urology, Norfolk \& Norwich University Hospital NHS Foundation Trust, Colney Lane, Norwich, NR4 7UY, UK

\begin{abstract}
Introduction/Aims: We have studied the natural history of Peyronie's disease with particular reference to erect (functional) penile length. To our knowledge this has so far not been documented.

Methods: 21 men with stable Peyronie's deformity were followed up for 6 months when no active treatment was being offered. By inducing an artificial erection objective assessments of penile length and deformity were made on day 1 and at six months.

Results: Measurable shortening in straight penile length was noticed in 6/21 (28.5\%) of men (Mean: $0.92 \mathrm{~cm})$, lengthening in $8 / 21(38.09 \%$ ) of men (Mean: $0.81 \mathrm{~cm}$ ), while no change was noticed in the remaining $7(33.3 \%)$.

Angulation increased (worsened) in 5 (23.8\%), decreased (improved) in 11 (52.38\%) and showed no change in 5 (23.8\%). There was no association between change in angle of deformity and change in penile length.

Conclusions: In Peyronie's disease, a progressive reduction in penile length is noticed in up to a third of patients with stable disease and this is not necessarily associated with deteriorating angle of deformity.
\end{abstract}

Keywords: Peyronie's disease, Natural history, functional length.

\section{INTRODUCTION}

With changing social attitudes an increasing number of men with Peyronie's disease are seeking advice and treatment for their deformity. Whilst urologists appreciate the need to counsel men before performing corrective surgery, there is little information for patients concerning the natural history of the disease should it be left untreated. The few published series [1,2] consist of a heterogeneous patient population and lack objective serial measurements. We hereby report the natural history of the disease, which we have observed, longitudinally with accurate measurements of erect penile length and deformity.

\section{METHODS}

Twenty-one men with treatment-naïve Peyronie's disease, which had been clinically stable for at least 6 months, were studied. None of them reportedly suffered from erectile dysfunction (ED) before or during the study period. The mean age was 60 yrs. (s.d. 10.5) and the mean duration of symptoms was 32.3 months (s.d. 28.0). Fourteen men had dorsal penile curvature and 7 had a dorso-lateral curvature with a solitary plaque along the dorsal and dorsolateral aspect of the mid-penile shaft respectively but none of the men had multiple or satellite plaques. All men were given an artificial erection by intracavernosal injection of PGE1 $(20 \mu \mathrm{g})$ with a uniformly satisfactory erectile response. 15 minutes post-injection, erect penile lengths (both straight and curved) were actually measured on the

*Address correspondence to this author at the Department of Urology, Norfolk \& Norwich University Hospital NHS Foundation Trust, Colney Lane, Norwich, NR4 7UY, UK;

Tel: 00441603286776; Fax: 00441603287884;

Emails: chitalenorwich@aol.com, sudhanshu.chitale@nnuh.nhs.uk individual subject as a distance from the tip of the glans penis to the pubis using rigid and flexible rulers. The angle of deformity was accurately measured using geometric protractors and these findings were documented with a clinical photograph and archived. Penile girth was not documented in this small series, as we did not come across any cases with complex curvatures such as hourglass deformities. The same operator repeated similar measurements six months later.

We used precise and properly validated techniques of measurements for this study. We validated our technique of measuring the erect penile length (straight \& curved) by testing it out (on a curved object) amongst a group of 11 health care professionals (HCP) and found a very small margin $(1.4 \%-2.35 \%)$ of error in measuring curved and straight lengths respectively, which proved insignificant for all practical purposes. We, therefore, relied upon measurements taken by a single HCP. Mean change in IIEF (International index of erectile function) was recorded over the 6-month study period but no partner data was available for the study population.

All men underwent penile ultrasonography $(7.5 \mathrm{Mhz}$ small parts probe) at both visits to ensure that none of the plaques were calcified and that the location of the plaques was on the same side as that of the curvature, but plaque dimensions were not specifically recorded.

\section{RESULTS}

The mean straight erect penile length for the 21 men on day one was $11.24 \mathrm{~cm}$ whereas the mean curved erect penile length was $14.39 \mathrm{~cm}$. Six months later, mean straight erect length for these men was $11.28 \mathrm{~cm}$ and mean curved length was $14.55 \mathrm{~cm}$. Over the six months (6/12), the erect straight 


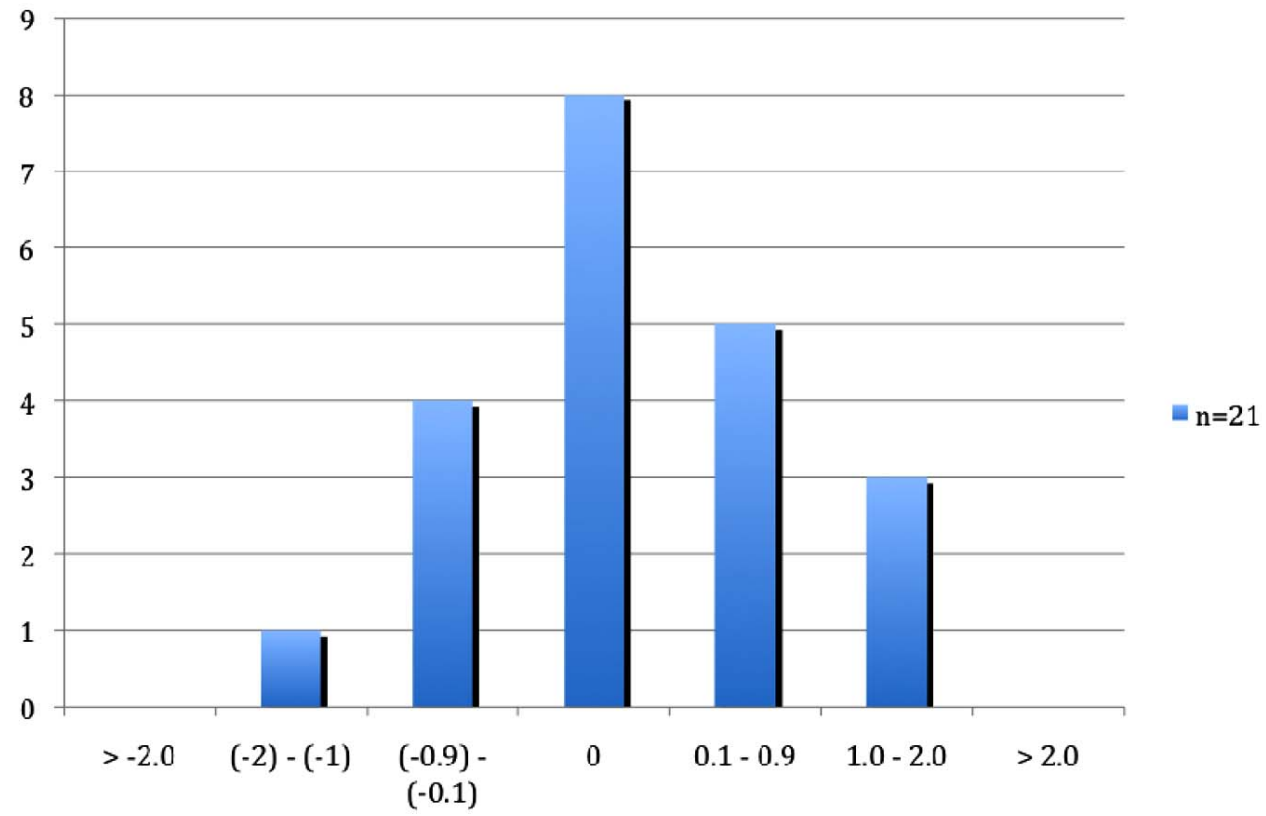

Fig. (1a). No. of pts. with change in straight length in $\mathrm{cm}(\mathrm{n}=21)$.

penile length decreased in 6 men $(28.5 \%)$ by a mean of 0.92 $\mathrm{cm}$, increased in 8 men $(38.09 \%)$ by a mean of $0.81 \mathrm{~cm}$ and was unchanged in the remaining 7 men (Fig. 1a).

The curved erect penile length decreased in 5 men $(23.8 \%)$ by a mean of $0.5 \mathrm{~cm}$, increased in 8 men $(38.09 \%)$ by a mean of $0.75 \mathrm{~cm}$ and remained unchanged in 8 men $(38.09 \%)$ (Fig. 1b). The mean change in the straight erect and curved erect lengths over six months was $0.048 \mathrm{~cm}$ and $0.16 \mathrm{~cm}$ respectively (Figs. 2a, b). Any change in length, however small, was considered a 'change' when no such change was expected in these patients long in to the inactive/ chronic phase. Moreover, the accuracy of measurements was validated prior to the study.

Over the same observation period, penile curvature increased in $5(23.8 \%)$, decreased in $11(52.38 \%)$ and remained unchanged in $5(23.8 \%)$. Tables 1 and 2 show the comparison between the change in straight/curved penile lengths in relation to the change in the angle of penile curvature revealing a predictable change in curved length in $8 / 21$ and a change in angle in 12/21 men and an opposite change in length in $8 / 21$ and a change in angle in $5 / 21$ with no change in length in $5 / 21$ and no change in angle in $4 / 21$

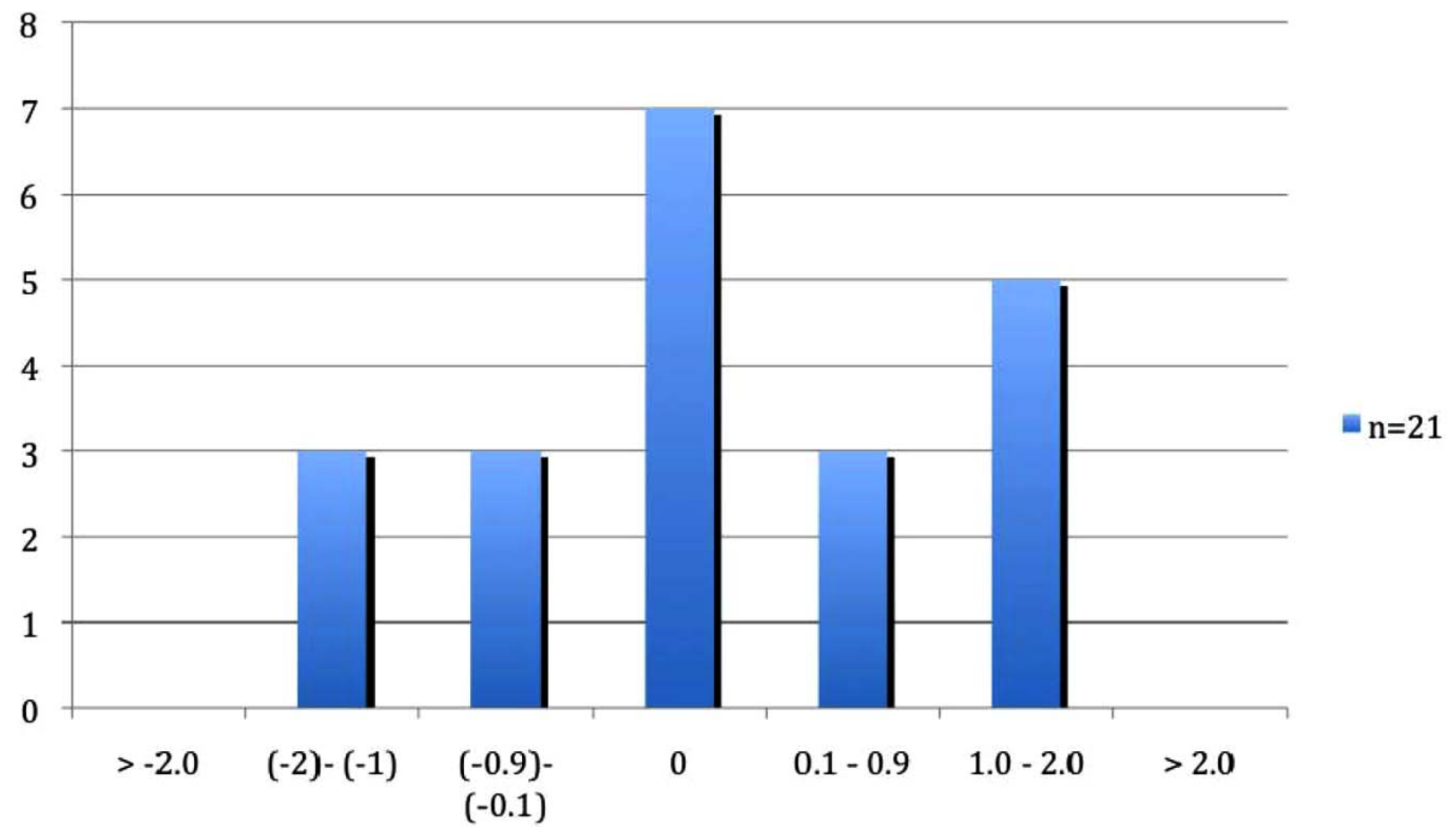

Fig. (1b). No. of pts. with change in curved length in $\mathrm{cm}(\mathrm{n}=21)$. 


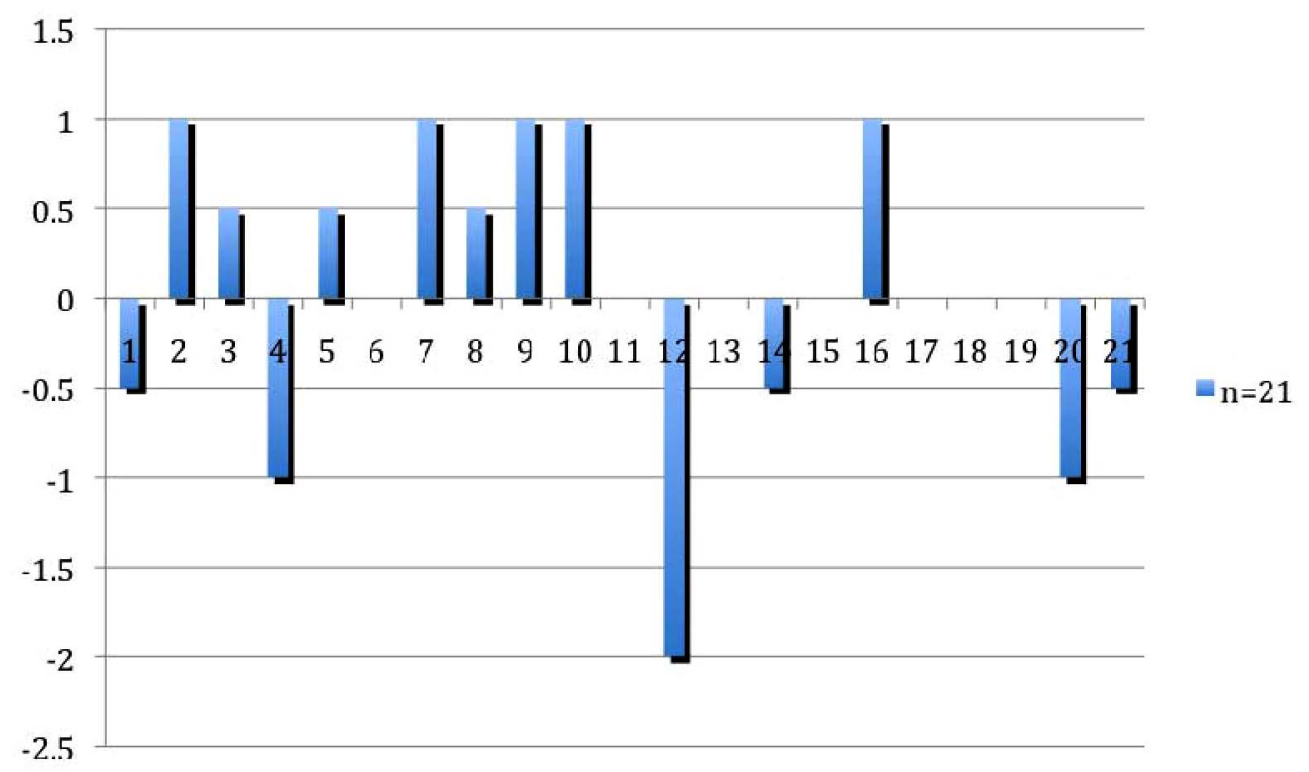

Fig. (2a). Change in straight lengths in $\mathrm{cm}(\mathrm{n}=21)$.

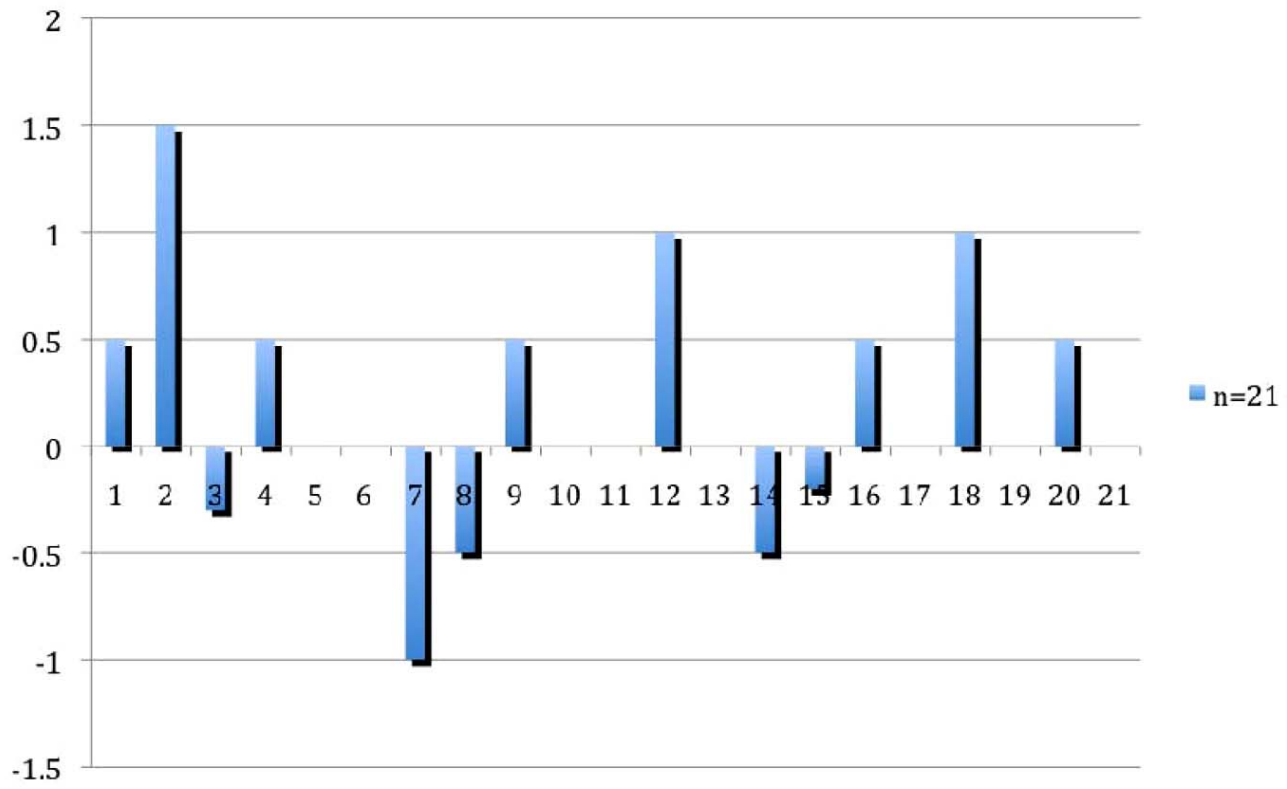

Fig. (2b). Change in curved lengths in $\mathrm{cm}(\mathrm{n}=21)$.

men. In 19/21 men there was no change in plaque dimensions or texture. In 2 men there was a marginal increase in plaque size.

Mean change in IIEF was $0.1 \pm 3.32$ over the study period.

\section{DISCUSSION}

Despite the occasional observational case series [1-6] reported in the literature, little is known about the natural history of Peyronie's disease. However, all these case series have varying drawbacks, as they have either had very small numbers [1-3] or have only been based on quality of life (QoL) questionnaires [4] or focussed on assessing relationship between age, co-morbidities [5], sonographic characteristics of the plaque and progression/stability of the disease [6]. Although flaccid penile length has been measured [7] to study natural history, to date there are no studies of sequential measurements of erect penile length and deformity. Our study looked at the natural history of PyD with reference to erect/functional penile length, which to our knowledge, has not been studied so far. There have been conflicting reports $[1-3,8-10]$ about the extent of natural resolution expected with Peyronie' deformity and the time it takes for the process to be complete as well as the appropriate timing and type of surgical intervention, all of which have significant implications on the preoperative 
Table 1. Comparison Between Change in the Straight Length and Change in the Angle of Curvature

\begin{tabular}{|c|c|c|c|c|}
\hline Str. Length Angle & Shortening (6) & Lengthening (8) & Unchanged (7) & Total (21) \\
\hline \hline Worse & 02 & 02 & 01 & 03 \\
\hline Better & 04 & 05 & 03 & 12 \\
\hline Unchanged & 00 & 01 & 04 \\
\hline
\end{tabular}

Table 2. Comparison Between Change in the Curved Length and Change in the Angle of Curvature

\begin{tabular}{|c|c|c|c|c|}
\hline Cur. Length & Shortening (5) & Lengthening (8) & Unchanged (8) & Total (21) \\
\hline \hline Angle & & & 00 & 06 \\
\hline Worse & 01 & 03 & 01 & 08 \\
\hline Better & 04 & 00 & 07 & 07 \\
\hline Unchanged & 00 & 03 & 06 \\
\hline
\end{tabular}

counselling and postoperative outcomes and their possible mismatch with patient expectations.

We observed a tendency to progressive shortening over a period of six months in nearly a third of our patients and although in a third the erect length remained unchanged; in the remaining third, there were signs of improvement at the end of $32.3+6=38.3$ months (s.d. 28). The angle of deformity and the curved erect penile length showed a change in the direction as predicted/hypothesized in $12 / 21$ $(57.2 \%)$ and $8 / 21(38.1 \%)$ patients respectively with a total of $11 / 21(52.38 \%)$ showing an ongoing improvement even after the disease had been allegedly stable for over six months. This finding has important implications on the success of surgical correction and the extent to which it could be attributed to simply natural resolution. It raises further doubts about the role of intervention in PyD $[10,11]$.

This study highlights the fact that penile shortening, in addition to being a known consequence of surgical correction of Peyronie's deformity; is also expected as a sequelae of natural progression of this disease and is likely to continue into the chronic phase long after the disease has been supposedly stable for sometime ( $>6$ months). Whilst it is common practice to delay surgery until the degree of deformity has been stable for several months, most urologists rely on the patient's observation to establish stability. A few units actually perform objective measurements of angulation $[6,7,12]$ but not the erect penile length, which equally matters to the patient. While it is imperative that patients are warned about the risk of shortening following penile corrective surgery, it is also important that they are made aware of the natural history of disease progression with worsening or continuing of shortening in the postoperative years.

This unpredictable pattern and unexpected behaviour also underlines the fact that we do not yet completely understand the behaviour of the Peyronie's plaque during the course of its natural evolution over a long term. A three-dimensional projection of the plaque exerting variable forces/torques on the configuration of the penile corporal cylinders in a circumferential manner could be responsible for the hitherto ill understood disease pathology and the lack of association between the angle of deformity and the penile length. Proteonomic profiling of $\mathrm{PyD}$ and understanding protein expression in PyD with its clinical implications will help us manage PyD better in future [13].

\section{LIMITATIONS OF OUR STUDY}

This study, a small pilot study, has some inherent limitations with large variation in the duration of symptoms within a small study population and a short period of observation/follow up; making it difficult to ascertain whether the change in length/curvature noted was a function of duration of symptoms. A study incorporating a larger number of patients with PyD observed and objectively assessed over longer period of time would help further understand the natural evolution of this disease. This would help prognosticate the impact of change in penile length on the severity of deformity and the choice/limitations of treatment options as well as the possible treatment outcomes in the light of a protracted course taken by the natural evolution of the disease as noted in our series. It would also be useful to measure both the flaccid stretched penile length as well as erect functional penile length to explore the relationship between anatomical shortening and functional shortcomings posed by the penile shortening in PyD which was not studied in the present series. Based on the observations made in this pilot, we aim to conduct a further study, recruiting a larger number of patients with a longer follow up that would enable better understanding of PyD. In this future extended study, we aim to measure both the flaccid and erect penile lengths and accurately document the plaque size to study correlation between the plaque shrinkage, deterioration in the angle of deformity and progressive/ongoing penile shortening as a part of the natural evolution of PyD over a longer time frame (up to 2 yrs.).

\section{CONCLUSION}

Erect penile length (straight/curved) may continue to decrease or increase in a large majority of patients with PyD and may show an unpredictable pattern due to variations in the plaque configuration and development. Peyronie's plaque may continue to evolve long into the chronic phase in a significant majority thereby raising doubts about the clear- 
cut demarcation described between active and inactive/chronic phase. Further research is required to understand the behaviour of the plaque and its implications on the natural history of PyD, understanding of which will help clinician and patients alike, make an informed decision regarding long term management of this perplexing disease entity.

\section{REFERENCES}

[1] Williams JL, Thomas GG. The natural history of Peyronie's disease. J Urol 1970; 103(1): 75-6.

[2] Ashworth A. Peyronie's disease. Proc Roy Soc Med 1960; 53: 6924.

[3] Kato R, Sato Y, Adachi H, et al. [Long-term clinical course of Peyronie's disease treated conservatively] Nippon Hinyokika Gakkai Zasshi 1997; 88(5): 571-5.

[4] Gelbard MK, Dorey F, James K. The natural history of Peyronie's disease. J Urol 1990; 144(6): 1376-9.

[5] Grasso M, Lania C, Blanco S, Limonta G. The natural history of Peyronie's disease. Arch Esp Urol 2007; 60(3): 326-31.
[6] Bekos A, Arvaniti M, Hatzimouratidis K, Moysidis K, Tzortzis V, Hatichristou D. The natural history of Peyronie's disease: an ultrasonography-based study. Eur Urol 2008; 53(3): 644-50.

[7] Mulhall JP, Schiff J, Guhring P. An analysis of the natural history of Peyronie's disease. J Urol 2006; 175(6): 2115-8.

[8] El-Sakka Al, Yasin AA. Conservative treatment for Peyronie'sdisease: an update. Expert Opin Pharmacother 2008; 9(1): 53-63.

[9] Bella AJ, Perelman MA, Brant WO, Lue TF. Peyronie's disease (CME): J Sex Med 2007; 4(6): 1527-38.

[10] Mira JG. Is it worthwhile to treat Peyronie's disease? Urology 1980; 16(1): 1-6.

[11] Carson CC. Potassium para-aminobemnzoate for the treatment of Peyronie's disease: is it effective? Tech Urol 1997; 3(3): 135-9.

[12] Ohbshalom M, Milhall J, Guhring P, Parker M. Measurement of penile curvature in Peyronie's disease patients: comparison of three methods. J Sex Med 2007; 4(1): 199-203.

[13] Domes T, De Young L, O'Gorman DB, Gan BS, Bella AJ, Brock G. Is there a role for proteomics in Peyronie's isease? J Sex Med 2007; 4(Pt. 1): 867-77. 